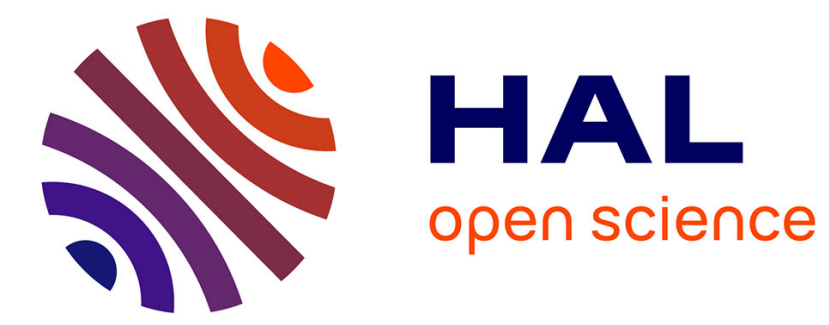

\title{
Politiques commerciales et de la concurrence
}

Patrick Messerlin

\section{To cite this version:}

Patrick Messerlin. Politiques commerciales et de la concurrence. Revue Economique, 1995, 46 (3), pp.717-726. 10.3406/reco.1995.409681 . hal-01009844

\section{HAL Id: hal-01009844 \\ https: / hal-sciencespo.archives-ouvertes.fr/hal-01009844}

Submitted on 18 Jun 2014

HAL is a multi-disciplinary open access archive for the deposit and dissemination of scientific research documents, whether they are published or not. The documents may come from teaching and research institutions in France or abroad, or from public or private research centers.
L'archive ouverte pluridisciplinaire HAL, est destinée au dépôt et à la diffusion de documents scientifiques de niveau recherche, publiés ou non, émanant des établissements d'enseignement et de recherche français ou étrangers, des laboratoires publics ou privés.

\section{(이) $\$$}

Distributed under a Creative Commons Attribution - NonCommercial - NoDerivatives| 4.0 


\title{
Persée
}

\author{
http://www.persee.fr
}

\section{Politiques commerciales et de la concurrence.}

\author{
Patrick A. Messerlin \\ Revue économique, Année 1995, Volume 46, Numéro 3 \\ p. 717 - 726
}

Voir l'article en ligne

\section{Avertissement}

L'éditeur du site " PERSEE » - le Ministère de la jeunesse, de l'éducation nationale et de la recherche, Direction de l'enseignement supérieur, Sous-direction des bibliothèques et de la documentation - détient la propriété intellectuelle et les droits d'exploitation. A ce titre il est titulaire des droits d'auteur et du droit sui generis du producteur de bases de données sur ce site conformément à la loi n`98-536 du 1 er juillet 1998 relative aux bases de données.

Les oeuvres reproduites sur le site «PERSEE » sont protégées par les dispositions générales du Code de la propriété intellectuelle.

Droits et devoirs des utilisateurs

Pour un usage strictement privé, la simple reproduction du contenu de ce site est libre.

Pour un usage scientifique ou pédagogique, à des fins de recherches, d'enseignement ou de communication excluant toute exploitation commerciale, la reproduction et la communication au public du contenu de ce site sont autorisées, sous réserve que celles-ci servent d'illustration, ne soient pas substantielles et ne soient pas expressément limitées (plans ou photographies). La mention Le Ministère de la jeunesse, de l'éducation nationale et de la recherche, Direction de l'enseignement supérieur, Sous-direction des bibliothèques et de la documentation sur chaque reproduction tirée du site est obligatoire ainsi que le nom de la revue et- lorsqu'ils sont indiqués - le nom de l'auteur et la référence du document reproduit.

Toute autre reproduction ou communication au public, intégrale ou substantielle du contenu de ce site, par quelque procédé que ce soit, de l'éditeur original de l'oeuvre, de l'auteur et de ses ayants droit.

La reproduction et l'exploitation des photographies et des plans, y compris à des fins commerciales, doivent être autorisés par l'éditeur du site, Le Ministère de la jeunesse, de l'éducation nationale et de la recherche, Direction de l'enseignement supérieur, Sous-direction des bibliothèques et de la documentation (voir http://www.sup.adc.education.fr/bib/). La source et les crédits devront toujours être mentionnés. 


\title{
Politiques commerciale et de la concurrence
}

\author{
Patrick A. Messerlin*
}

\begin{abstract}
Cet article examine les problèmes posés par la coexistence, difficile, entre les politiques du commerce extérieur et de la concurrence, dans le contexte du GATT et de son héritière, l'Organisation mondiale du commerce (OMC). Elle montre que ces problemes, s'ils sont traditionnels, se posent en des termes nouveaux, particulièrement graves, à cause de la mise en couvre, par les États, de politiques commerciales à visée anticoncurrentielle. Cet article passe en revue les propositions actuelles pour résoudre ces difficultés, et offre une approche plus limitée, mais plus réaliste.
\end{abstract}

Classification JEL : F1, K2, L4

Cet article examine les problèmes posés par la coexistence, difficile, entre les politiques du commerce extérieur (politique commerciale) et de la concurrence. Il le fait dans un contexte précis : celui des négociations commerciales du GATT et de son héritière, l'Organisation mondiale du commerce (OMC).

La section 1 montre que ces problèmes ne sont pas des inconnus dans ce cadre : dès la Charte de La Havane, ils font l'objet de discussions et de décisions. Mais la section 2 montre que, depuis les années quatre-vingt, ces problèmes se posent en des termes nouveaux, particulièrement graves: la mise en cuvre, par les États, de politiques anticoncurrentielles, d'une façon de plus en plus délibérée. La section 3 passe en revue les propositions actuelles pour résoudre ces difficultés : leur caractéristique commune est de chercher refuge dans une loi mondiale de la concurrence ou la mise en cuvre « harmonisée » des lois nationales de la concurrence. La section 4 propose une approche plus limité : celle-ci repose sur une utilisation systématique de la politique de la concurrence comme garde-fou des instruments de politique commerciale et sur la progressive introduction de concepts de politiques de la concurrence lors de la mise en cuvre des instruments de politique commerciale.

\footnotetext{
*. Institut d'études politiques, 27, rue Saint-Guillaume, 75007 Paris.
} 


\section{UN PROBLÈME PRÉSENT DEPUIS LA CHARTE DE LA HAVANE : LA RÉPRESSION DES COMPORTEMENTS ANTICONCURRENTIELS DES FIRMES}

Comme le rappellent Lloyd et Sampson [1994], les négociations commerciales internationales ont, dès l'après-guerre, abordé les aspects de politique de la concurrence liés aux seuls comportements anticoncurrentiels des firmes.

Le chapitre V de la Charte de La Havane élaborée en 1946-1947 est tout entier consacré aux "pratiques restrictives" des entreprises, comme, entre autres, la fixation des prix, le partage des marchés, la mise en cuuvre de quotas de production, les restrictions en matière de développement de la technologie et certaines utilisations de brevets, marques déposées et autres droits de propriété intellectuelle.

La Charte inaugure trois traditions : elle ne concerne que la dimension internationale (les entraves à la concurrence des seules firmes domestiques ne sont pas concernées) ; elle est fondée sur une profonde suspicion à l'égard des firmes (et, corrélativement, la croyance en des Etats parfaitement informés et soucieux du seul bien-être collectif); et, enfin, elle ne cherche à créer qu'une soft law, c'est-à-dire des dispositions appliquées sur une base purement volontaire par les États et ne portant que sur des conditions de transparence, consultation et conciliation entre les gouvernements.

On retrouve ces trois aspects dans les principaux textes adoptés jusqu'à l'Uruguay Round.

Le texte initial du GATT ne contient aucune disposition similaire au chapitre V de la Charte : cela s'explique par le fait que le texte du GATT a été adopté en urgence, pour servir de support juridique au premier Round des baisses tarifaires déjà acceptées par les pays signataires. Entre 1954 et 1960, des négociations, dans le cadre du GATT, aboutissent à un accord ad hoc sur les cartels et les trusts internationaux. Cet accord établit des procédures de notification et consultation : celles-ci n'ont jamais été utilisées.

L'OCDE prend la relève avec une série de recommandations sur les pratiques anticoncurrentielles affectant le commerce international (1967, 1973, 1979 et 1986) et avec les directives sur les entreprises multinationales. Avec ces dernières, l'OCDE ne s'adresse plus aux seuls gouvernements : ces directives décrivent, pour les firmes concernées, des comportements à éviter, dans les domaines des acquisitions, de la prédation, du refus de vendre, de la discrimination et des prix de transferts.

En 1980, la CNUCED adopte, à son tour, un « Ensemble de principes et règles sur le contrôle des pratiques restrictives » applicable à toutes les transactions dans les biens et services. Cet ensemble qui ne constitue pas une obligation légale demande aux gouvernements de mettre en œuvre une politique de la concurrence (pour leurs entreprises privées et publiques).

Enfin, l'Uruguay Round a apporté son lot de références à la politique de la concurrence : dans l'accord sur les sauvegardes, dans le GATS (accord sur les services), dans l'accord sur les droits de proprieté intellectuelle et dans celui sur les mesures concernant les investissements liés au commerce, et enfin, sans doute l'innovation la plus spectaculaire, dans l'accord «plurilatéral » sur les marchés publics. Selon ce dernier, une firme s'estimant en butte à des pratiques 
anticoncurrentielles dans la mise en cuvre des procédures d'offre de marchés publics, a, pour la première fois dans le droit international, le soutien de cet accord plurilatéral (lié au GATT et à l'OMC) pour déposer une plainte directe auprès des tribunaux du pays concerné pour essayer d'obtenir gain de cause.

\section{UN PROBLÈME NOUVEAU DEPUIS LE TOKYO ROUND : L'ÉMERGENCE DES « POLITIQUES ANTICONCURRENTIELLES » DES ÉTATS}

Les années soixante-dix et surtout quatre-vingt changent progressivement les termes du problème. Elles révèlent le rôle croissant des États dans la création de limitations à la concurrence internationale. En particulier, les instruments récents de la politique commerciale, notamment les actions antidumping, constituent des politiques « anticoncurrentielles » délibérées en ce sens qu'ils permettent aux Etats de soutenir et de maintenir des comportements anticoncurrentiels de certaines firmes.

Ce nouvel aspect des relations entre politiques commerciale et concurrentielle est bien plus inquiétant que celui traité traditionnellement par les textes mentionnés dans la première section. En effet, des efforts de cartellisation ou de collusion des prix entre entreprises privées n'ont que des chances limitées de survie à long terme s'ils ne correspondent à aucune rationalité économique. Au contraire, une mesure antidumping imposée par un Etat peut assurer, pour de longues années, la survie d'un cartel trop faible pour survivre par lui-même ${ }^{1}$.

R.C. de Grey [1986] a été le premier, dans une étude faite à la demande de l'OCDE mais jamais rendue publique par celle-ci, à présenter les États comme des créateurs de cartels durables ou d'arrangements préjudiciables à la concurrence internationale. L'essentiel de son argumentation se concentre sur les limitations volontaires à l'exportation, particulièrement abondantes au début des années 1980, et dont le Tokyo Round avait été impuissant à limiter l'expansion.

Depuis le début des années quatre-vingt, les actions antidumping prennent le relais des limitations volontaires des exportations et deviennent le cour de ces politiques anticoncurrentielles (Messerlin, [1990]).

Quatre caractéristiques font que les actions antidumping se prêtent parfaitement à ce rôle des États «bras séculiers » de cartels ou d'ententes de prix qui auraient du mal à se créer et à survivre sans l'appui des autorités publiques. En premier lieu, les actions sont de facto à la disposition des seules firmes, donc disponibles pour celles qui veulent limiter la concurrence. En second lieu, les firmes plaignantes doivent représenter au moins $25 \%$ de l'industrie domestique, ce qui est à la portée des seules firmes ayant de larges parts de marché, ou d'une coalition de ces firmes. Les procédures d'enquête sont tellement biaisées qu'elles assurent un succès hors du commun : seulement $5 \%$ des actions anti-

1. Ceux qui connaissent les résultats de l'Uruguay Round soutiendront que l'adoption de la clause des cinq ans (sunset clause) limite cette affirmation. En fait, il y a de nombreux arguments suggérant que cette disposition est d'un impact limité. 
dumping concluent à l'absence de dumping. Les mesures prises sont toutes de type collusif : soit ouvertement (c'est le cas des engagements de prix ou de quantités), soit de facto (c'est le cas des droits de douane qui, de par les termes mêmes des règlements antidumping, ne peuvent pas être « absorbés » par les firmes étrangères même si celles-ci sont suffisamment efficientes pour le faire).

Messerlin [1990] montre, cas par cas, les relations entre les actions antidumping (et anticoncurrentielles) et les enquêtes des autorités de la concurrence pour manquement aux règles de concurrence du traité de Rome. Au cours des années quatre-vingt, environ un quart des actions antidumping reflètent un quart des cas soumis à la direction générale de la Concurrence (DG-IV). Ce chiffre global peut se décomposer en quatre éléments. En premier lieu, la moitié des cas examinés pendant les années quatre-vingt au titre de l'article 85: un (actions concertées entre entreprises menant à des accords horizontaux ou à des cartels) traite des mêmes produits, marchés et firmes que des cas antidumping initiés dans la même période (ou de produits, marchés et firmes très proches). En second lieu, il en est de même pour un petit nombre des cas examinés au titre de l'article 86 (abus de position dominante). En troisième lieu, six cas traitant de systèmes de distribution créant des obstacles à l'exportation ou à l'importation et isolant des marchés d'États membres du reste des marchés communautaires (et mondiaux) ont fait aussi l'objet d'actions antidumping. Enfin, cinq des quarante-trois cas dans lesquels la DG-IV a accepté une certaine forme de coopération entre les entreprises communautaires (une exemption des règles de concurrence) pour une durée limitée dans le temps ont aussi des prolongements en termes d'actions antidumping.

En fait, les effets économiques des actions antidumping s'analysent fort bien en termes de raising rivals' costs (Salop et Scheffman [1983]). Les actions antidumping réduisent les ventes des firmes accusées de dumping en augmentant leurs couts de trois manières : les droits antidumping augmentent directement les coûts de production des usines étrangères ; ils réduisent les taux d'utilisation des capacités de production des concurrents, décourageant par là même les investissements futurs ; enfin, en limitant l'accès au marché (importateur), les actions antidumping réduisent la capacité d'éliminer les risques associés aux marchés, augmentant par là le risque moyen et la variabilité du risque associés aux exportations. Ces deux derniers aspects sont particulièrement nuisibles si les entreprises étrangères affectent leurs usines à des marchés d'exportations spécifiques (dont le marché protégé par les mesures antidumping est une part importante).

Tous ces effets des actions antidumping, manifestement contraires à ce que la politique concurrentielle recherche, méritent trois remarques. Tout d'abord, les effets décrits ci-dessus correspondent aux trois principaux composants de la valeur des actifs d'une firme. Ce faisant, ils réduisent la valeur actualisée des usines des firmes étrangères prises dans les actions antidumping. Du coup, une firme plaignante peut trouver une action antidumping doublement bénéfique : non seulement, celle-ci protège son marché, mais encore elle tend à faire baisser la valeur de l'usine étrangère, rendant cette dernière moins coûteuse à l'achat, tout en ouvrant la possibilité de laisser cette usine aussi efficiente qu'avant l'action antidumping, une fois l'usine achetée par le plaignant (lequel pourra faire supprimer les droits antidumping). Ensuite, les stratégies de raising rivals' costs sont, sans doute, plus « efficaces»-c'est-à-dire plus couteuses pour les 
firmes étrangères visées et/ou moins couteuses pour les entreprises nationales que celles consistant à baisser les prix ou à s'engager dans des guerres de prix. Enfin, ces stratégies ne cherchent pas nécessairement à éliminer les firmes étrangères du marché d'importation : ces firmes peuvent demeurer présentes, mais avec des coûts élevés en sorte qu'elles jouent alors le rôle de « soutien » des prix. En particulier, interdire l'absorption des droits antidumping par les firmes étrangères ne laisse à ces dernières d'autre solution que d'investir directement dans le pays importateur - avec le risque de supporter des conts de production plus élevés et de devenir, par nécessité, un défenseur de la protection existante.

\section{LA RECHERCHE DES DISCIPLINES CONCURRENTIELLES INTERNATIONALES}

Devant cette évolution préoccupante, l'approche proposée de nos jours est de renforcer les politiques concurrentielles en leur donnant le caractère international qu'elles n'ont pas à présent. Dans cette perspective, le premier problème à résoudre est, comme le souligne Graham [1994], celui de la dose de «supranationalité ${ }^{1}$ ». L'expérience de la Communauté montre que ce problème peut être résolu : un "noyau dur» de lois communautaires et sa mise en œuvre coexistent avec les lois des Etats membres et leurs applications. En même temps, les développements les plus récents montrent que l'expérience communautaire est particulière. L'extension de ce noyau dur à des pays proches de la Communauté, comme les pays d'Europe centrale, se révèle un processus assez long et difficile, même si le soutien politique pour une telle extension est acquis.

Aussi, les solutions apportées se répartissent-elles en trois groupes. En premier lieu, il y a des tentatives pour rédiger des lois mondiales de la concurrence (Hauser et Schöne [1994]). Le caractère supranational de ces lois est réduit par deux moyens : ces lois visent des comportements très précis, et elles sont fondées sur le respect de « seuils » quasi automatiques pour définir les entraves à la concurrence (par exemple, des seuils de parts de marché).

Une seconde solution est celle de l'harmonisation des lois nationales sur un modèle. En général, cette harmonisation ne concerne que le noyau dur des dispositions à prendre. L'illustration de cette approche est celle des lois de la concurrence en Europe centrale.

Enfin, il y a des solutions reposant sur un emploi coordonné des lois nationales de la concurrence. Cette utilisation suppose la « reconnaissance mutuelle » des lois de la concurrence de chacune des parties. Celle-ci peut se faire sur la base de comités « négatifs » ou " positifs ». Sous la règle du negative comity, un pays s'impose de ne pas appliquer ses lois de la concurrence dans le cadre de la juridiction des autres pays. Sous la règle du positive comity, un pays assiste les

1. Les soft laws internationales (décrites plus haut) ont montré leur stérilité : elles n'ont entraîné aucune application. Il faut donc des dispositions juridiques entraînant des sanctions, en cas de non-respect. 
autorités en charge de la concurrence d'un autre pays, en «mettant à sa disposition » les pouvoirs d'enquête de ses propres autorités.

C'est dans ce cadre de positive comity que Hoekman et Mavroidis [1994] ont suggéré d'aborder les problèmes antidumping. Partant du principe que seul le dumping «stratégique» (Willig [1992]) pourrait, éventuellement, justifier la mise en cuvre d'actions antidumping, ils suggèrent qu'avant toute ouverture d'actions antidumping les autorités en charge de la concurrence dans le pays d'origine des exportateurs vérifient la contestabilité de ce marché d'origine : les actions antidumping dans le pays importateur ne seraient engagées que si cette contestabilité n'était pas démontrée.

Cependant, malgré les apparences, même les solutions fondées sur la notion de positive comity soulèvent de grosses difficultés qui naissent des contraintes liées au respect d'une stricte confidentialité des informations.

\section{UNE APPROCHE RECENTRÉE SUR LA POLITIQUE COMMERCIALE}

Une approche en termes de disciplines concurrentielles internationales tend à négliger trois aspects : l'autre moyen de l'échange international que sont les investissements directs internationaux, les limites de l'action des autorités en charge de la concurrence, et les possibilités de « capturer » les lois existantes de la concurrence. Si elle peut prendre en compte le premier aspect sans trop de difficultés (au moins en partie), il lui est, en revanche, difficile de tenir en compte des deux derniers aspects.

C'est pourquoi une approche recentrée sur la politique commerciale per se apparaît souhaitable.

Le premier aspect négligé concerne l'interaction entre investissements directs étrangers (à destination ou en provenance de l'étranger) et politiques commerciales. Cet aspect est appelé à jouer un rôle très important dans la décennie qui vient, à cause de la concurrence accrue entre pays en développement (et pays développés), pour attirer les capitaux étrangers. Cette concurrence fait que les pays en développement tendent à accorder des conditions de plus en plus privilégiées aux firmes investissant chez eux. Ces privilèges prennent souvent la forme de décisions de politique commerciale, car c'est souvent la seule façon encore à la portée de ces pays de "payer " les investisseurs : les firmes qui investissent dans un pays obtiennent un accès privilégié au marché du pays, par le biais de préférences dans des quotas existants, de structures tarifaires taillées sur mesure, etc. Un excellent exemple de ces problèmes est donné par les investissements des firmes européennes automobiles dans les pays d'Europe centrale (il est quelque peu ironique de constater que le degré de concentration sur ces marchés n'est pas loin de celui existant en 1989). En ignorant ce type de problèmes, l'approche en termes de disciplines concurrentielles internationales tend à n'introduire que des disciplines étroitement liées au commerce international stricto sensu, ce qui paraît une approche discutable sur le plan de l'analyse économique (créer des instruments de portée limitée risque de créer des distorsions dans l'économie nationale). 
En second lieu, il faut être conscient des limites de l'action des autorités en charge de la concurrence. Ces dernières sont, dans tous les pays, dans la mouvance du pouvoir exécutif. En tant que telles, elles ne peuvent ignorer et, a fortiori, aller à l'encontre de ce que d'autres parties de ce même pouvoir exécutif ont pu décider. Cela inclut, bien évidemment, les décisions en matière de politique commerciale, donc les décisions en matière de limitations volontaires des exportations ou d'antidumping. Une excellente illustration de ce type de situations (plus fréquentes qu'on ne le croit) est le comportement des autorités britanniques de la concurrence à l'égard des limitations volontaires des exportations signées entre les constructeurs japonais et britanniques d'automobiles : ces autorités n'ont pu intervenir contre un accord pourtant présenté comme purement privé (dont les gouvernements simplement « connaissaient » l'existence).

Enfin, les lois de la concurrence ne sont pas, elles-mêmes, d'une robustesse sans limites : elles peuvent être "capturées " par des interêts protectionnistes (et plus elles seront en première ligne, plus le risque sera grand). Il est utile de rappeler que les premiers règlements antidumping sont nés des lois de la concurrence, l'exemple le plus célèbre (mais non le seul) étant la section 73 de l'U.S. Tariff Act de 1894. En d'autres termes, ce sont des dispositions bien précises de lois de la concurrence qui ont été totalement retournées contre leur objectif initial : la section 73 du Wilson Tariff Act complétait la section 2 du Sherman Antitrust Act de 1890 sur les politiques de prix prédatrices. Ces dispositions ont encore des traits essentiels des lois de la concurrence : la section 73 ne soumet pas le prédateur à des droits antidumping visant à compenser le dumping, mais à des amendes et/ou à des peines de prison. Aussi, peu de cas antidumping seulement ont été ouverts sous ces lois (un seul sous la section 73) ${ }^{1}$. La capture de ces dispositions concurrentielles par des intérêts protectionnistes se produit en 1904 au Canada qui est le premier pays à adopter une loi antidumping sans référence à des comportements économiques précis sur des marchés ${ }^{2}$. Désormais, le dumping est simplement défini comme la situation où le prix pratiqué par l'exportateur sur son marché domestique est supérieur à celui que le même exportateur demande dans le pays importateur ${ }^{3}$. En outre, cette simple inégalité arithmétique en termes de prix, sans aucune recherche sur le comportement des firmes impliquées, est désormais «punie » d'une taxe antidumping perçue à la frontière et dont le seul objet est d'annuler la différence calculée. L'approche canadienne devient rapidement à la mode : au début des années trente, tous les grands pays ont adopté des dispositions juridiques équivalentes.

1. Ce faible nombre de cas reflète l'impact de l'obligation faite de tenir compte des intérêts des consommateurs, mais aussi le rôle de contraintes proprement juridiques (l'absence de dispositions formelles concemant l'extra-territorialité dans les dispositions antidumping, et le caractère pénal des lois antitrust américaines).

2. Viner [1921, p. 193] note combien les motifs de la loi canadienne étaient de fournir un « fusible » pour les pressions protectionnistes croissantes.

3. La définition donnée du prix domestique est la suivante : « la valeur normale (fair market value) de l'article similaire vendu sur le marché domestique au cours d'opérations commerciales normales ". Les principaux concepts de l'article VI du GATT sont, tous, déjà là. 
Ce détournement des dispositions antidumping peut paraître lié à des aspects dépassés de la vie ou de l'analyse économiques. Il n'en est rien, comme l'illustrent les deux exemples suivants. Le premier est fourni par Mattoo et Mavroidis [1994] : les effets attendus du remplacement des quotas nationaux dans l'automobile par un quota européen ont été largement réduits par des « règles de concurrence d'exception » en matière de distribution automobile. Le second exemple est lié à la libéralisation de services dont l'infrastructure a été (est) détenue par un monopole (public). L'exploitation de cette infrastructure a souvent été associée à la production de certains services « annexes » dans lesquels le monopole prolonge sa position dominante. Quand la liberalisation des services est engagée, elle engendre, en général, l'émergence de nouveaux services, partiellement en concurrence avec les services annexes traditionnels du monopole. Celui-ci peut alors se trouver dans une situation de «dumping stratégique » : dominant sur ses marchés traditionnels, il peut faire pression sur les marchés des nouveaux services, mettant en péril les firmes entrant sur ces marchés. Ce type de situation est, à bien des égards, si proche de ceux examinés dans des cas antidumping que les dispositions des lois de la concurrence traitant de ces aspects peuvent se révéler aisées à être « capturées » par des groupes protectionnistes.

Ces considérations incitent à se tourner vers une approche reposant sur deux principes : une mise en ouvre plus systématique de la politique de la concurrence comme garde-fou des instruments de la politique commerciale, et une progressive introduction de concepts de politique de la concurrence dans l'emploi de ces instruments. En d'autres termes, cette approche repose sur une moindre utilisation des instruments de politique de la concurrence et sur une amélioration renforcée des instruments de politique commerciale.

Une mise en cuvre systématique et séquentielle de la politique de la concurrence permettrait de mettre les actions antidumping sous la menace explicite d'actions concurrentielles. Cette option séquentielle présente plusieurs avantages.

Le premier est de reposer sur des précédents historiques. Le premier cas est celui de Matsushita-Zenith aux Etats-Unis. Après avoir usé de tous les instruments de politique commerciale disponibles (limitations volontaires des exportations, antidumping, antisubvention), Zenith dépose une plainte contre vingt et une firmes japonaises de TV-couleur : elle accuse ces dernières de s'entendre pour monopoliser le marché américain par une politique de bas prix, financée par des prix élevés au Japon, eux-mêmes possibles grâce à la fermeture du marché japonais à la concurrence étrangère. Le second précédent est celui d'Extramet, une petite firme française, consommatrice d'un produit (le calcium métal) fabriqué exclusivement par Péchiney en Europe. Extramet a estimé que la première action antidumping déclenchée par Péchiney avait pour seul objet d'éliminer des concurrents (d'Union soviétique et de Chine) pour conserver une position dominante, avec la capacité d'en abuser. Il est intéressant de noter que, dans les deux cas, les autorités en charge de la concurrence n'ont pas soutenu les mesures antidumping prises. La Cour Suprême américaine a débouté Zenith, estimant que les entreprises japonaises avaient réduit leurs prix simplement pour affronter la concurrence sur le marché américain (Blair, Fesmire et Romano [1991]). Ét la Cour de justice européenne (qui, jusqu'au cas Extramet, n'avait statué que sur de purs aspects de procédure dans les plaintes d'entreprises victimes de mesures antidumping) a déclaré que les aspects de concurrence 
devaient être pris en compte lors des décisions des mesures antidumping, reconnaissant, implicitement, le rôle anticoncurrentiel de ces mesures.

Le second avantage de cette approche est qu'elle réduit au minimum les problèmes d'extra-territorialité, un risque certain dans les cas liés aux affaires antidumping. Cela favorise l'adoption unilatérale de cette approche, une attitude cohérente avec le principe, bien connu depuis Ricardo, du bienfait d'une libéralisation unilatérale de l'économie.

Le dernier avantage est que, lorsque les réglementations antidumping seront devenues moins attrayantes pour les firmes concurrentes des importations, ces entreprises chercheront et trouveront (certainement) d'autres instruments de protection. Soumettre ces nouveaux instruments au même contrôle de la politique de concurrence sera plus facile et plus naturel à obtenir si cela a déjà été un fait acquis pour les actions antidumping. En d'autres termes, la politique de la concurrence reste la politique horizontale qu'elle doit être.

Ces différents points renforcent la cohésion avec l'idée centrale du GATT : celle de n'exiger des pays qu'une application non discriminatoire des principes gouvernant un pays. Si un pays tient aux règles de concurrence, il doit pouvoir les appliquer en toutes circonstances : en ce cas, les autorités en charge de la concurrence doivent pouvoir faire apparaître une analyse des bénéfices et des coûts de la protection, et demander que cette dernière, si elle est finalement imposée, soit obtenue par l'instrument le moins coûteux en termes économiques. En d'autres termes, les autorités de la concurrence seraient davantage en charge de réduire le coût de la protection, laissant au gouvernement son rôle, en la matière, de décideur en dernier ressort.

Reste que ce premier élément serait insuffisant sans des progrès significatifs en ce qui concerne les instruments de politique commerciale. Ceux-ci peuvent porter sur deux aspects.

En premier lieu, il convient d'adopter de meilleurs moyens de protection per se. Cela peut être illustré par les dispositions antidumping du traité de Rome. Dans la partie consacrée aux " règles communes ", le chapitre sur les règles de concurrence contient l'article 91 , consacré aux pratiques de dumping dans les échanges intra-CEE. La disposition « boomerang » de l'article $91: b$ est particulièrement remarquable : elle limite l'emploi de mesures antidumping par la possibilité, pour le pays importateur, de ré-exporter les produits accusés de dumping vers le pays d'origine, en totale exemption de barrières.

En second lieu, l'impact négatif des instruments de protection peut être grandement réduit par la mise en ouvre de concepts directement tirés des lois de la concurrence. Ce point peut facilement être illustré par un exemple. Les actions antidumping reposent sur la notion de "produit similaire». Celle-ci permet des manipulations accroissant, de façon considérable, la probabilité de trouver du dumping et l'impact des mesures prises. Substituer à cette notion le concept de "marché pertinent " tiré des lois de la concurrence constituerait un important pas en avant dans la remise en ordre des politiques anticoncurrentielles de ces dernières années. 


\section{REFERENCES BIBLIOGRAPHIQUES}

BlaIR R.D., Fesmire J.M. et Romàno R.E. [1991], «An Economic Analysis of Matsushita ", The Antitrust Bulletin, été, p. 355-381.

GRAHAM E.M. [1994], « Competition Policy and the New Trade Agenda », mimeo, juin, OECD Secretariat.

DE GREY R.C. [1986], « Trade and Competition Policies », mimeo, OECD Secretariat.

HAUSER H. et SCHÖNE R. [1994], «Is there a Need for International Competition Rules », Aussenwirtschaft, Heft II/II, p. 205-222.

HoEKMAN B.M. et MAVROIDIS P.C. [1994], « Antitrust-Based Remedies and Dumping in International Trade », Policy Research, Working Paper 1347, aott, The World Bank.

LLOYD P. et SAMPSON G. [1994], « Competition and Trade Policy : Identifying the Issues after the Uruguay Round », mimeo, septembre, GATT Secretariat.

MATTOO A. et MAVROIDIS P.C. [1994], « The EC-Japan Consensus on Cars : Interaction between Trade and Competition Policy », mimeo, GATT Secretariat.

MESSERLIN P.A. [1990], «Antidumping Regulations or Pro-Cartel Laws, The EC Chemical Cases ", The World Economy, décembre, p. 465-492.

SalOP S.C., SCHEFFMAN S.C. [1983], «Raising Rivals' Costs », American Economic Review, p. 267-271.

VINER J. [1921], Dumping : A Problem in International Trade, reprinted by A.M. Kelley, 1966, Reprints of Economic Classics, New York. 